\title{
Bacillus Calmette-Guérin immunisation at birth and morbidity among Danish children: A prospective, randomised, clinical trial
}

\author{
Lisbeth Marianne Thøstesen $^{\mathrm{a}, *}$, Thomas Nørrelykke Nissen ${ }^{\mathrm{b}}$, Jesper Kjærgaard ${ }^{\mathrm{c}}$, Gitte Thybo Pihl ${ }^{\mathrm{a}}$, \\ Nina Marie Birk ${ }^{\mathrm{b}}$, Christine Stabell Benn ${ }^{\mathrm{d}, \mathrm{e}}$, Gorm Greisen ${ }^{\mathrm{f}}$, Poul-Erik Kofoed ${ }^{\mathrm{a}, \mathrm{g}}$, Ole Pryds ${ }^{\mathrm{b}}$, \\ Henrik Ravn ${ }^{\mathrm{d}, \mathrm{e}}$, Dorthe Lisbeth Jeppesen ${ }^{\mathrm{b}}$, Peter Aaby ${ }^{\mathrm{h}}$, Lone Graff Stensballe ${ }^{\mathrm{c}, \mathrm{d}}$ \\ a Department of Paediatrics, Kolding Hospital, Skovvangen 2-8, DK-6000 Kolding, Denmark \\ b Department of Paediatrics 460, Copenhagen University Hospital, Hvidovre, Kettegaard Allé 30, DK-2650 Hvidovre, Denmark \\ c The Child and Adolescent Clinic 4072, Juliane Marie Centret, Rigshospitalet, Copenhagen University Hospital, Blegdamsvej 9, DK-2100 Copenhagen Ø, Denmark \\ ${ }^{d}$ Research Center for Vitamins and Vaccines (CVIVA), Bandim Health Project, Statens Serum Institut, Artillerivej 5, DK-2300 Copenhagen S, Denmark \\ e OPEN, Institute of Clinical Research, University of Southern Denmark/Odense University Hospital, DK-5000 Odense, Denmark \\ ${ }^{\mathrm{f}}$ Neonatal Department, Juliane Marie Centret, Rigshospitalet, Copenhagen University Hospital, Blegdamsvej 9, DK-2100 Copenhagen Ø, Denmark \\ ${ }^{g}$ Institute of Regional Health Research, University of Southern Denmark/Odense University Hospital, Odense, Denmark \\ ${ }^{\mathrm{h}}$ Bandim Health Project, Statens Serum Institut, Artillerivej 5, DK-2300 Copenhagen S, Denmark
}

\section{A R T I C L E I N F O}

\section{Article history:}

Received 27 January 2015

Received in revised form 7 April 2015

Accepted 8 April 2015

Available online 18 April 2015

\section{Keywords:}

Bacillus Calmette-Guérin

BCG vaccination

Children

Heterologous immunity

Non-specific effects of vaccines

Randomised clinical trial

\begin{abstract}
A B S T R A C T
Background: Studies from low-income countries report positive non-specific effects of early Bacillus Calmette-Guérin (BCG) immunisation on childhood health and survival. Neonatal immunisation with BCG may prime the immune system and offer partial protection against other infectious and possibly allergic diseases. The potential clinical value of these nonspecific effects has not yet been examined in a large randomised trial in high-income countries.

Methods: The Danish Calmette Study is a multicentre randomised clinical trial conducted between October 2012 and November 2015. Within the first 7 days of life, infants were randomly assigned to intra-dermal vaccination with BCG or no intervention. At 3 and 13 months of age structured telephone interviews and clinical examinations of the children were conducted. In a subgroup of children blood samples were drawn and stool samples collected at age 4 days, 3 and 13 months. Thymus index was assessed by ultrasound in a subgroup at randomisation and at 3 months. The primary study outcome is hospitalisation within the first 15 months of life as assessed in Danish health registers. Secondary outcomes include infectious disease hospitalisations, wheezing, eczema, use of prescribed medication, growth, development, thymus index, T- and B-cell subpopulations assessed by flow cytometry, in vitro cytokine responses and specific antibody responses to other vaccines. Adverse reactions were registered.
\end{abstract}

Abbreviations: ASQ Ages and Stages Questionnaire; BCG, Bacillus Calmette-Guérin; C. alb, Candida albicans; cm, centimetre; CPR, Central Person Register; DSMB, Data Safety and Monitoring Board; E. coli, Escherichia coli; e-crf, electronic case report form; g, grams; IgE, immunoglobulin E; IFN- $\gamma$, interferon gamma; IL, interleukin; LPS, lipopolysaccharide; MMR, measles, mumps, rubella; Pentavalent vaccine, vaccine against diphtheria, tetanus, Bordetella pertussis, polio and Haemophilus influenza type B; PHA, phytohaemagglutanin; Pneumococcal conjugate vaccine, 13-valent vaccine against Streptococcus pneumoniae; SCORAD, scoring atopic dermatitis; SSI, Statens Serum Institut; S. pneu, Streptococcus pneumoniae; Tc, T-cytotoxic cells; Th, T-helper-cell; TNF- $\alpha$, tumor necrosis factor alpha.

* Corresponding author. Tel.: + 4524774826.

E-mail addresses: lmje@dadlnet.dk (L.M. Thøstesen), tnnissen@gmail.com (T.N. Nissen), jekjaergaard@gmail.com (J. Kjærgaard), gitte.thybo.pihl@rsyd.dk (G.T. Pihl), ninabirk@gmail.com (N.M. Birk), cb@ssi.dk (C.S. Benn), Gorm.Greisen@regionh.dk (G. Greisen), Poul.Erik.Kofoed@rsyd.dk (P.-E. Kofoed), pryds@dadlnet.dk (O. Pryds), hjn@ssi.dk (H. Ravn), Dorthe.Lisbeth.Jeppesen@regionh.dk (D.L. Jeppesen), p.aaby@bandim.org (P. Aaby), lone.graff.stensballe@regionh.dk, lgn@ssi.dk (L.G. Stensballe). 
Discussion: With participation of 4184 families and more than $93 \%$ adherence to clinical follow-up at 3 and 13 months, this randomised clinical trial has the potential to create evidence regarding non-specific effects of BCG vaccination in a high-income setting.

(C) 2015 The Authors. Published by Elsevier Inc. This is an open access article under the CC BY-NC-ND license (http://creativecommons.org/licenses/by-nc-nd/4.0/).

\section{Introduction}

The Bacillus Calmette-Guérin vaccine (BCG) is administered to infants in more than 100 countries to protect against the most severe forms of tuberculosis [1]. Due to a declining incidence of tuberculosis, BCG was withdrawn from the Danish Childhood Immunisation Program in the 1980s.

Many observational studies from low-income settings have reported positive non-specific effects of neonatal BCG immunisation on childhood health and survival [2-5]. One study from West Africa found reduced sensitisation to allergens among BCG-immunised children [5]. Recently, two randomised trials among low-birth-weight children observed a significantly lower mortality in the group BCG-immunised at birth than those who received the usual delayed BCG [6,7]. Morbidity and mortality due to infectious diseases in early infancy are high in West Africa while tuberculosis is very uncommon in infancy. Therefore, a protective mechanism has to be non-specific.

During the past three decades eczema and asthma have become the most common chronic diseases among children in high-income countries [8]. Research suggests that a skewed immunologic stimulation, possibly caused by increased hygiene and fewer infections, may be part of the explanation for the increased prevalence of these diseases [9]. BCG strongly stimulates the immune system, resulting in an interferon gamma (IFN- $\gamma$ ) response [10] that may counterbalance the Thelper-cell (Th)-2 response found in atopic individuals. It has therefore been hypothesised that BCG could have a protective effect on atopic diseases, but results from several retrospective studies have been inconsistent [11-14]. According to a metaanalysis from 2014 BCG vaccination might have exerted a protective effect against non-atopic asthma, although it did not protect against allergic sensitisation [15]. Apart from a small randomised trial from the Netherlands [16], no randomised trials of BCG's effect on allergic diseases have been conducted. The Dutch trial found significantly reduced use of medication for eczema among the BCG-immunised children [16].

In the present trial we aimed to test the effect of BCG on hospitalisation and morbidity including allergic diseases in high-income settings in a large clinical trial with patientrelevant outcomes. Our main hypothesis was that neonatal BCG vaccination would be associated with a $20 \%$ reduction in hospitalisation during the first 15 months of life.

\section{Methods/design}

The Danish Calmette Study is a multicentre randomised clinical trial with 1:1 allocation of newborns within 7 days of birth to either BCG vaccination or no intervention. The inclusion criteria were gestational age of at least 32 weeks, a birth weight of at least $1000 \mathrm{~g}$, and a signed consent form from the parents. Exclusion criteria were maternal intake of immune modulating medicine during pregnancy or signs of severe illness or major malformation in the newborn. Only parents fluent in Danish were included, as questionnaires in Danish were used.

\section{Setting}

From October 2012 to November 2013 all parents planning to give birth at Rigshospitalet, Hvidovre Hospital, and Kolding Hospital received a letter in the 2nd or 3rd trimester of pregnancy with information about the Danish Calmette Study and an invitation to participate in the study. Invited parents were contacted by phone and offered further information either on the phone or, on request, at a personal meeting. We obtained ethical permission to inform the women by phone.

\section{Interventions}

Within 7 days of birth participants were randomised to either the intervention group (BCG immunisation) or to the control group using an electronic case report form (e-crf). Randomisation was stratified by sex, hospital and gestational age ( $\geq 37$ weeks of gestation versus $<37$ weeks). Block sizes were 2-4-6 in random order. Twins and triplets were allocated to the same randomisation group based on the sex of the first born child. The random allocation sequence was generated by the e-crf shortly before the vaccination and was concealed to all study staff until the intervention was assigned. Specially trained midwives and study health professionals at the three trial sites assigned participants to intervention and vaccinated children in the BCG group intra-dermally with BCG vaccine, SSI strain 1331, in the standard dose of $0.05 \mathrm{ml}$ in the upper, lateral part of the left shoulder. The children randomised to the control group were not given a placebo vaccine and the parents were not blinded to the intervention because no other injection can mimic the typical reaction following a BCG vaccination.

The English product summary of BCG from SSI can be found on the website http://www.immune.org.nz/sites/ default/files/resources/

SSI\%20BCG\%20vaccine\%20data\%20sheet.pdf.

Before randomisation of their child the parents received written and oral information about the most common adverse reactions after BCG vaccination (Table 1 ).

Table 1

Adverse reactions after BCG vaccination.

$\begin{aligned} \text { Uncommon }(>1 / & \text { Systemic: Headache, fever. } \\ 1000,<1 / 100) & \text { Local: Enlargement of regional lymph node } \\ & >1 \mathrm{~cm} \text {. Ulceration with a discharging ulcer at the } \\ & \text { site of injection. } \\ & \text { Systemic: Disseminated BCG complications as } \\ & \text { osteitis or osteomyelitis. Allergic reactions, } \\ & \text { including anaphylactic reactions. } \\ & \text { Local: Suppurative lymphadenitis, abscess } \\ & \text { formation. }\end{aligned}$


In case of suspected adverse reactions other than the expected redness, swelling, skin sore, swollen regional lymph nodes and a scar, the parents were instructed to contact the study staff as soon as possible, so the case could be reported and medical advice be given and, if needed, the child referred to clinical control and treatment. The Data Safety and Monitoring Board (DSMB) of the Danish Calmette Study regularly evaluated the patterns of adverse reactions.

\section{Outcomes}

The primary outcome of the Danish Calmette Study is hospitalisation in the first 15 months of life. The secondary outcomes include infectious disease hospitalisations, atopic dermatitis, asthmatic bronchitis, use of prescribed medication, development, anthropometrics measured at 3 and 13 months, immunological outcomes ( $\mathrm{T}$ - and B-cell subpopulations assessed by flow cytometry and cytokine production in stimulated whole blood) measured at 4 days of age, 3 and 13 months and thymus volume as measured by ultrasound scanning (thymus index) and the ratio between thymus volume and infant body weight (thymus/weight index) measured at inclusion and at 3 months. We also investigated which factors affect parents' decision concerning participation in the Danish Calmette Study and whether their experience with the BCG vaccination influenced their attitude towards future vaccinations.

\section{Data collection}

\subsection{Background information}

A structured telephone interview was conducted prior to randomisation focusing on demographics and risk factors such as atopic disposition and parental smoking habits. All telephone interviews, including the later mentioned follow-up interviews, were performed by specially trained study staff. All members of the study staff had a medical background as either doctors, nurses, midwives, medical students or midwife students.

\subsection{Registry-based data}

Hospitalisations were identified using the Danish National Patient Registry [17]. All Danish citizens have a unique social security number (CPR) that can be used to link data at an individual level. The Danish Register of Medicinal Product Statistics contains CPR-number-based information about sale of all prescribed medicines in Denmark. The included children were followed in these registries until 15 months of age for hospitalisations, total medication use, including use of antibiotics, anti-asthmatics, and topical medication against eczema.

\subsection{Clinical and paraclinical data}

\subsubsection{At inclusion}

Thymic scans: At the time of randomisation, just before vaccination, thymus index (a volume estimate) and thymus/ weight index (the ratio between thymus volume and infant body weight) were assessed using a transsternal ultrasonic method [18] on a subpopulation of 300 children (thymus cohort), including the 150 children who delivered a blood sample (see below).

\subsubsection{4-day follow-up}

Blood samples: At Hvidovre Hospital blood samples were obtained from 150 children (blood sample cohort), 75 children from each randomisation group, 4 days after randomisation. The samples were used for immunological analyses, including total leukocyte count, assessment of $\mathrm{T}$ - and B-cell subpopulations (recent thymic emigrants, naive cells, central memory cells, effector memory cells, late differentiated cells, Th17 cells, Tc17 cells, regulatory B-cells, regulatory T-cells, chronic activated cells, apoptic cells) measured by sixcolour flow cytometry in whole blood and examination of the production of cytokines (tumor necrosis factor (TNF)- $\alpha$, interleukin (IL)-1 $\beta$, IL-6, IFN- $\gamma$, IL-10, IL-17, IL-22) using whole blood stimulated in vitro with other pathogens, RPMI (medium alone), lipopolysaccharide (LPS), phytohaemagglutanin (PHA), Escherichia coli (E. coli), Streptococcus pneumonia (S. pneu), Candida albicans (C. alb), and BCG. Stool samples for microbiome analyses were obtained from a subset of participants in the blood sample cohort.

\subsubsection{3-month follow-up}

Structured telephone interviews were conducted at age 3 months to obtain parent-reported data on illness episodes, vaccinations, anthropometrics and nutrition. At the end of the telephone interview the e-crf revealed a few supplementary questions to parents of the BCG-vaccinated children about the reaction at the vaccination site and the information received about adverse reactions. All children were invited for a clinical examination at the study facility.

The clinical examination was conducted by specially trained study staff (doctors and nurses) and included: anthropometrics (weight, height and head circumference), heart and lung auscultation, and examination for eczema and wheeze. In case of atopic dermatitis, the Scoring Atopic Dermatitis (SCORAD) form was used [19]. Parents were instructed not to reveal the randomisation group of their child when interviewed on the phone and when they attended the clinical examination. Before performing the clinical examination we asked the parents to cover the typical site of vaccination using a plaster. At Hvidovre Hospital blood samples were drawn from the blood sample cohort and the immunological analyses were repeated. Stool samples were obtained from a subset of participants in the blood sample cohort and thymus index and thymus/weight index were assessed in the thymus cohort.

\subsubsection{3-month follow-up}

The structured telephone interview from the 3-month follow-up was repeated, supplemented with questions concerning the use of healthcare services. To measure the children's psychomotor development, their parents were asked to fill out a standardised, validated online Ages and Stages Questionnaire (ASQ) [20]. The children were invited for a clinical examination.

The clinical examination assessed the same outcomes as at 3 months of age, supplemented with measuring of skin folds, abdominal circumference and mid upper arm circumference (MUAC). At the very end of the last clinical examination the 
length and width of the BCG scar was measured after the parents had removed the plaster.

At Hvidovre Hospital blood samples were drawn from the blood sample cohort and in addition from 150 other children, thus providing 300 blood samples, 150 from each randomisation group. The immunological analyses were repeated, supplemented by analyses for specific antibody responses to other vaccines (Diphteria, tetanus, Bordetella pertussis, Haemophilus influenzae type b and S. pneu) and analyses for allergic sensitisation (specific immunoglobulin E). Stool samples were obtained from a subset of participants in the blood sample cohort. All children included at Kolding Hospital were invited to have a blood sample drawn for analysis for allergic sensitisation.

\subsubsection{Data collection specifically for premature children}

As a safety measure we followed the premature children (born at gestational age 32 weeks +0 days to 36 weeks +6 days ) more intensively. Psychomotor development at 6, 13 and 22 months after birth was assessed by means of ASQ.

\section{Sample size consideration, power calculation}

At least $20 \%$ of the child population in Denmark are hospitalised during the first year of life [21]. Sample size estimates were based on 95\%-confidence intervals and 90\% power. To detect a $20 \%$ protection against hospitalisation during the first 15 months of life, 3972 infants needed to be included (1986 BCG-immunised and 1986 non-BCGimmunised). With an incidence of $5 \%$ of eczema in the child population below 15 months of age, it was necessary to include 4230 infants (2115 in each group) to be able to detect a $40 \%$ protection against eczema. We therefore aimed at including 4300 newborns. Sub-analyses on sub-groups of participants or less frequent events may not be adequately powered.

\section{Monitoring and rules of termination}

The trial was supervised by the Good Clinical Practice Units in the Capital Region and in the Region of Southern Denmark. The study was monitored by an independent DSMB with three members who evaluated the study status, patterns of adverse reactions, and the distribution of background information and outcomes in the two groups of randomisation. The trial was to be terminated if BCG had a significantly negative effect $(p<0.01)$ or a significantly positive effect $(p<0.001)$ on physician consultations as obtained through the interviews and/or on parental report of eczema.

\section{Statistical methods}

Comprehensive statistical analysis plans were developed for the primary as well as for secondary outcomes; these plans were deposited with the DSMB before the analyses were commenced. Data were analysed by "intention-to-treat" and "per protocol" and adjusted for the stratified randomisation design. The "per protocol"-analyses excluded children, who did not follow their randomisation groups. Thus, both children randomised to BCGvaccination but not BCG-vaccinated, and children randomised to no BCG-vaccination but BCG-vaccinated outside the Calmette Study were excluded from the "per protocol"-analyses. We analysed outcomes in Cox proportional hazards model, so hospitalisations occurring before the date of enrolment into the trial were ignored. Children were followed until 15 months of age, corresponding to the age of MMR immunisation (mumps, measles and rubella) according to the Danish Childhood Immunisation Program.

The possible effect of BCG was analysed overall from 0 to 15 months, but also analysed corresponding to the periods when childhood vaccines are given during the first year of life: at 3, 5 and 12 months of age. At these time points Danish children are due to receive the pentavalent vaccine (against diphtheria, tetanus, Bordetella pertussis, polio and Haemophilus influenzae type B) and the pneumococcal conjugated vaccine (vaccine against 13 strains of $S$. pneu). Hence, it is possible to test if the potential BCG effect is modified by subsequent vaccines. We hypothesise the effect of BCG immunisation to be most pronounced in the first 3 months of life. The randomised trials of BCG from West Africa [6,7] have shown that the positive effects of BCG in boys occur already in the first week of life while they occur later for girls (authors' unpublished data). Thus, we tested the possible effects of BCG and their timing separately for boys and girls. P-values less than $5 \%$ were considered statistically significant and all tests were two-sided.

\section{Registration number}

The trial was approved by the Danish Data Protection Board (J.no. 2009-41-4141), the Committees on Biomedical Research Ethics (J.no. H-3-2010-087), and the Danish Medicines Agency (J.no. 2612-4356. EudraCT 2010-021979-85. Protocol 2009323). The study was registered at www.ClinicalTrials.gov with trial registration number NCT01694108.

\section{Economy}

The study was initiated and planned by the researchers and funded by the three involved hospitals and by the Danish National Research Foundation (DNRF108). Supplementary funding was granted by other non-commercial foundations. The BCG vaccine was produced by Statens Serum Institut, Copenhagen, Denmark, and bought by the Danish Calmette Study. The study participants were insured by the Patient Insurance Act. There was no remuneration for participation in the Danish Calmette Study.

\section{Steering Group}

The Steering Group consists of representatives from the three inclusion sites, from Research Centre for Vitamins and Vaccines and from Bandim Health Project. The Steering Group is responsible for the data and blood samples collected.

\section{Discussion}

During 14 months in 2012-2013 a total of 4184 families were included in the Danish Calmette study. The crude adherences at the clinical examination at 3 and 13 months of age were above $93 \%$. More than $85 \%$ completed the ASQ 


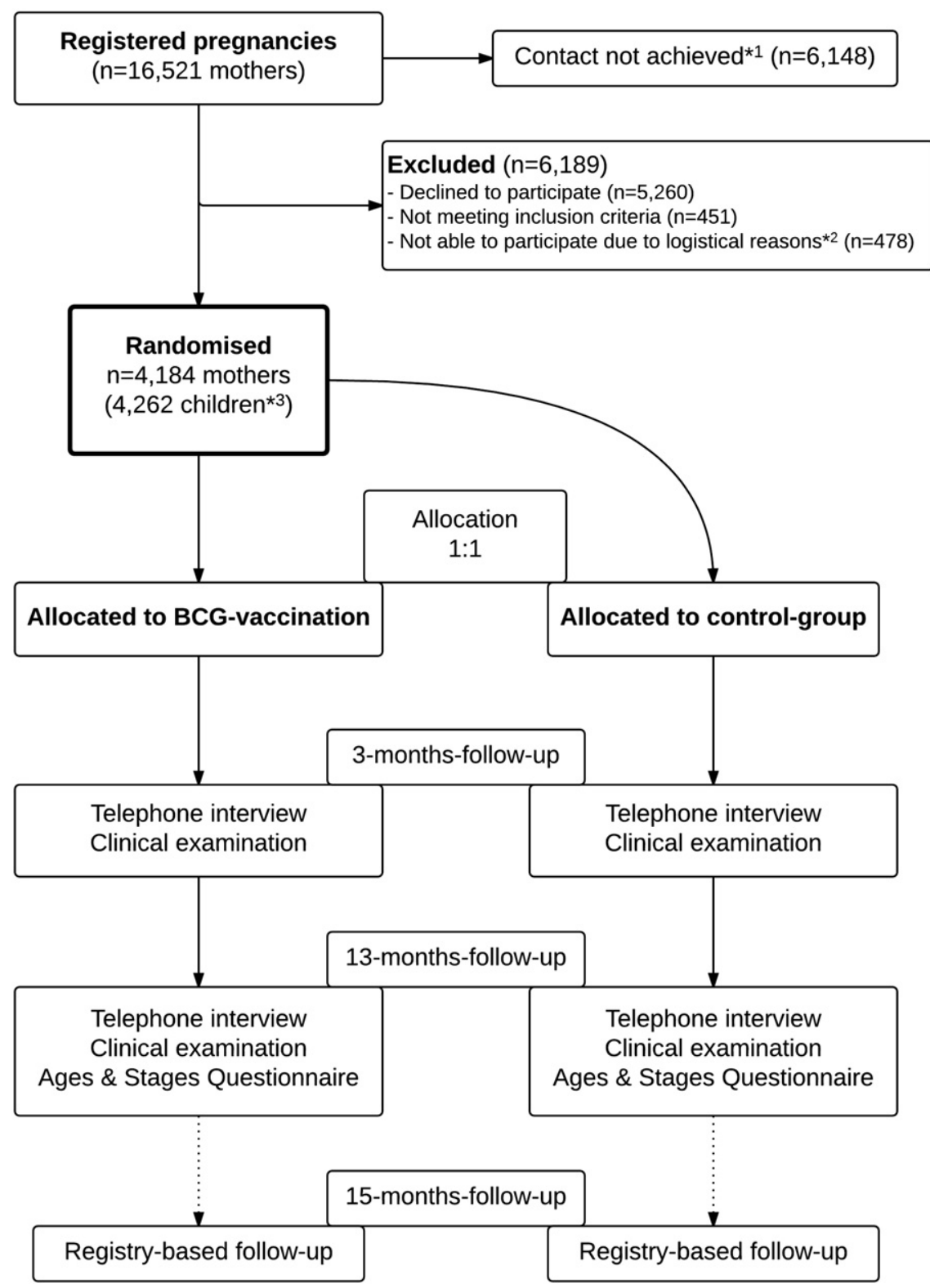

Fig. 1. Inclusion into the Danish Calmette Study and plan for the follow-up. ${ }^{* 1}$ : Not all invited women could be contacted in spite of at least five telephone calls or due to the telephone number being unknown or due to lack of staff (mainly initially in the study period). ${ }^{* 2}$ : Some children were not randomised within their first 7 days of life due to big work-load at the maternity ward or because the family by mistake was discharged before randomisation. Some children were born at other hospitals and thus not randomised. ${ }^{* 3}: 4105$ children were born as singletons, 154 as twins, and three as triplets. Twins and triplets were randomised to the same group.

questionnaire at 13 months of age. Since the Danish health registers are population based, the registry-based follow-up included $100 \%$ of the study population (Fig. 1 ).

Collecting the main outcome data from national registries reduces the risk of bias as data were registered by people outside the study group, independent of the exposure.

If BCG vaccination at birth can reduce early childhood morbidity in high-income settings, this may have significant public health and socio-economic impact, both by improving the quality of life for the infants and their families and by reducing healthcare costs.

\subsection{Trial status}

The Danish Calmette Study is a large multicenter randomised clinical trial in Denmark with more than 4000 participants and an adherence at 1 year follow-up of more than 93\%. The recruitment stopped November 2013 and the clinical follow-up ended January 2015. Data from Danish health registers were obtained in 2015.

\subsection{Competing interests}

We declare that we have no conflict of interests. 


\subsection{Authors' contributions}

LGS conceived the idea of the Danish Calmette Study. The Steering Group of the Danish Calmette Study (LGS, PA, CSB, GG, OP and PEK) wrote the Danish protocol of the study with statistical contributions from HR and with later additions from DLJ. LMT, TNN, JK, GTP and NMB participated substantially in the data collection and in the revision of the protocol. The drafting of this manuscript was led by LMT, with major contributions from the other authors. All authors read and approved the final manuscript.

\section{Acknowledgements}

We thank the many families for their participation and loyalty, and the Calmette study staff for their continuing commitment. We thank the DSMB-members Frank Shann, Professor of Paediatric Intensive Care Medicine, Melbourne; Per Kragh Andersen, Professor of Biostatistics, Copenhagen; and Jørn Olsen, Professor of Epidemiology, Aarhus (chair).

\section{References}

[1] Fine PEM, Carneiro IAM, Milstien JB, Clements CJ. Issues relating to the use of BCG in immunization programmes. A discussion document. WHO Department of Vaccines and Biologicals; 1999 (http://whqlibdoc.who.int/ hq/1999/WHO_V\&B_99.23.pdf).

[2] Garly ML, Martins CL, Bale C, Balde MA, Hedegaard KL, Gustafson P, et al. BCG scar and positive tuberculin reaction associated with reduced child mortality in West Africa. A non-specific beneficial effect of BCG? Vaccine 2003;21(21-22):2782-90.

[3] Roth A, Gustafson P, Nhaga A, Djana Q, Poulsen A, Garly ML, et al. BCG vaccination scar associated with better childhood survival in GuineaBissau. Int J Epidemiol 2005;34(3):540-7. http://dx.doi.org/10.1093/ije/ dyh392.

[4] Roth A, Sodemann M, Jensen H, Poulsen A, Gustafson P, Weise C, et al. Tuberculin reaction, BCG scar, and lower female mortality. Epidemiology 2006;17(5):562-8. http://dx.doi.org/10.1097/01.ede.0000231546.14749. ab.

[5] Aaby P, Shaheen SO, Heyes CB, Goudiaby A, Hall AJ, Shiell AW, et al. Early BCG vaccination and reduction in atopy in Guinea-Bissau. Clin Exp Allergy 2000;30(5):644-50.

[6] Aaby P, Roth A, Ravn H, Napirna BM, Rodrigues A, Lisse IM, et al. Randomized trial of BCG vaccination at birth to low-birth-weight children: beneficial nonspecific effects in the neonatal period? J Infect Dis 2011; 204(2):245-52. http://dx.doi.org/10.1093/infdis/jir240.

[7] Biering-Sorensen S, Aaby P, Napirna BM, Roth A, Ravn H, Rodrigues A, et al. Small randomized trial among low-birth-weight children receiving bacillus Calmette-Guerin vaccination at first health center contact. Pediatr Infect Dis J 2012;31(3):306-8. http://dx.doi.org/10. 1097/INF.0b013e3182458289.

[8] Mortz CG, Lauritsen JM, Bindslev-Jensen C, Andersen KE. Prevalence of atopic dermatitis, asthma, allergic rhinitis, and hand and contact dermatitis in adolescents. The Odense Adolescence Cohort Study on Atopic Diseases and Dermatitis. Br J Dermatol 2001;144(3):523-32.

[9] Strachan DP. Family size, infection and atopy: the first decade of the "hygiene hypothesis". Thorax 2000;55(Suppl. 1):S2-S10.

[10] Marchant A, Goetghebuer T, Ota MO, Wolfe I, Ceesay SJ, De Groote D, et al. Newborns develop a Th1-type immune response to Mycobacterium bovis bacillus Calmette-Guerin vaccination. J Immunol 1999;163(4):2249-55.

[11] Obihara CC, Beyers N, Gie RP, Potter PC, Marais BJ, Lombard CJ, et al. Inverse association between Mycobacterium tuberculosis infection and atopic rhinitis in children. Allergy 2005;60(9):1121-5. http://dx.doi.org/ 10.1111/j.1398-9995.2005.00834.x.

[12] Townley RG, Barlan IB, Patino C, Vichyanond P, Minervini MC, Simasathien T, et al. The effect of BCG vaccine at birth on the development of atopy or allergic disease in young children. Ann Allergy Asthma Immunol 2004; 92(3):350-5. http://dx.doi.org/10.1016/s1081-1206(10)61574-8.

[13] Gruber C, Kulig M, Bergmann R, Guggenmoos-Holzmann I, Wahn U. Delayed hypersensitivity to tuberculin, total immunoglobulin E, specific sensitization, and atopic manifestation in longitudinally followed early Bacille Calmette-Guerin-vaccinated and nonvaccinated children. Pediatrics 2001;107(3) E36.

[14] Singh M, Das RR, Kumar L, Kumar R. Bacille Calmette-Guerin vaccination is associated with lower prevalence of allergic diseases in Indian children. Am J Rhinol Allergy 2013;27(4):e107-12. http://dx.doi.org/10.2500/ajra. 2013.27.3940.

[15] Linehan MF, Nurmatov U, Frank TL, Niven RM, Baxter DN, Sheikh A. Does BCG vaccination protect against childhood asthma? Final results from the Manchester Community Asthma Study retrospective cohort study and updated systematic review and meta-analysis. J Allergy Clin Immunol 2014;133(3):688-95 [e14].

[16] Steenhuis TJ, van Aalderen WM, Bloksma N, Nijkamp FP, van der Laag J, van Loveren $\mathrm{H}$, et al. Bacille-Calmette-Guerin vaccination and the development of allergic disease in children: a randomized, prospective, single-blind study. Clin Exp Allergy 2008;38(1):79-85. http://dx.doi.org/ 10.1111/j.1365-2222.2007.02859.x.

[17] Andersen TF, Madsen M, Jorgensen J, Mellemkjoer L, Olsen JH. The Danish National Hospital Register. A valuable source of data for modern health sciences. Dan Med Bull 1999;46(3):263-8.

[18] Hasselbalch H, Jeppesen DL, Engelmann MD, Michaelsen KF, Nielsen MB. Decreased thymus size in formula-fed infants compared with breastfed infants. Acta Paediatr 1996;85(9):1029-32.

[19] Severity scoring of atopic dermatitis: the SCORAD index. Consensus Report of the European Task Force on Atopic Dermatitis. Dermatology 1993;186(1):23-31.

[20] Bricker D, Squires J. Ages \& Stages Questionnaires: a parent-completed, child-monitoring system. 2nd ed. Baltimore, MD: Paul H. Brookes Publishing Co; 1999.

[21] Folkesundhed SIf. Folkesundhedsrapporten. http://www.si-folkesundhed. dk/upload/kap_30_b\%C3\%B8rn.pdf; 2007. Accessed 13.03.2015. 